\title{
DISTINTOS NIVELES DE ANÁLISIS PARA EL ESTUDIO DEL CAMBIO CONCEPTUAL EN EL DOMINIO DE LA MECÁNICA
}

\author{
OLIVA, JOSÉ M. \\ Centro de Profesorado de Cádiz \\ E-mail: cnaveat@meditex.es
}

\begin{abstract}
SUMMARY
A quasi-experimental study on the conceptual change in mechanics, it has been accomplished following various levels of analysis. Eighty-five students of tenth grade of a public school completed two questionnaires on mechanics phenomena, before and after being taught. The obtained results showed the presence of qualitative and quantitative changes in the cognitive structure. These changes affected the specific ideas expressed through particular answers, as well as the most general frameworks on which those answers were based. From those results some implications for didactic interest and for the investigation are commented.
\end{abstract}

\section{PLANTEAMIENTO DEL PROBLEMA}

Los objetivos y métodos de los estudios sobre concepciones alternativas en ciencias han vivido algunos cambios importantes durante los últimos años. Se ha pasado de trabajos descriptivos dedicados a explorar las concepciones en distintos ámbitos a trabajos que investigan el nivel de estructuración y organización de las ideas a través de diversos fenómenos y dominios. Prueba de este cambio de interés lo tenemos en la atención despertada últimamente por los estudios sobre consistencia de las concepciones a través de distintos contextos y por aquellos otros que analizan la existencia de patrones de razonamiento generales que expliquen la gran variedad y diversidad de concepciones específicas catalogadas en distintos dominios.

En un sugerente artículo publicado hace unos años, Viennot (1985) apuntaba ya la posibilidad de que muchos de los argumentos que aportan los alumnos en sus respuestas fuesen debidos a la aplicación de reglas causales comunes a ellos. Si dos situaciones o tareas son caracterizadas como análogas, tenderá a aplicarse en ellas las mismas reglas de inferencia. Si son consideradas de distinta naturaleza, se aplicarán reglas también diferentes. Algo más tarde, Andersson (1986) ha planteado la necesidad de sistematizar el creciente número de ideas catalogadas y analizar la existencia o no de elementos comunes compartidos por esquemas explicativos existentes en diferentes dominios de conocimiento. Recientemente Monk (1995) ha propuesto un principio de estructuración para profundizar en las características de las concepciones en distintos ámbitos. Para Monk, este principio consiste en asumir que las diversas concepciones de un mismo sujeto no son entidades dispersas sino que se organizan y estructuran en sistemas de más alto nivel, siguiendo un símil, de forma semejante a cómo se organizan las estrellas en estructuras más complejas como son las galaxias.

El grado de estructuración que los autores más habituales han visto en las concepciones que han estudiado ha variado de unos casos a otros. La mayoría de ellos podrían situarse sobre un continuo entre dos polos o tendencias que han marcado el estatus asignado a las ideas de los alumnos. De un lado, están quienes piensan que el pensamiento es algo heterogéneo que mantiene un comportamiento fuertemente dependiente del contenido 
y del contexto. De otro, están quienes opinan que la mente humana es homogénea y consistente, siendo posible identificar estructuras de conocimiento universales que los sujetos aplicarían a la hora de razonar en dominios y contextos variados. En su visión más radical, esta idea de estructuración en el conocimiento tiene como referente a aquellos autores que consideran que los conceptos y nociones de los adolescentes en dominios específicos dependen de estructuras lógicas generales que son independientes del contenido. Desde esta posición se subordina el interés de las ideas particulares a la presencia o no de esas estructuras generales de las cuales dependerían. La tendencia actual de los trabajos es una evolución hacia un punto a medio camino entre uno y otro extremo.

En un trabajo anterior (Oliva, 1999a), hemos analizado el grado de estructuración de las concepciones sobre mecánica en alumnos de 14-16 años en una fase previa a la instrucción. Los datos obtenidos mostraron la existencia de un cierto grado de estructuración en las contestaciones, que podría catalogarse como intermedio entre el que establece el enfoque de las operaciones formales y el que predice el enfoque más fragmentario de las concepciones alternativas (Pozo et al., 1992). El grado de generalidad encontrado podría ser suficiente para postular la posibilidad de cambios en algunas concepciones -mediatizados en mayor o menor medida por la enseñanza recibida- una vez que se hayan cambiado otras con las que comparte algunos elementos de las estructuras implícitas. Y de ello se deduciría que superar las concepciones erróneas desde el punto de vista de la ciencia escolar conllevaría algo más que un cambio local en contenidos específicos. Comportaría un cambio en las teorías implícitas en las que subyacen las respuestas y contestaciones, esto es, en las estructuras mentales que con carácter más o menos general se encuentran tras ellas. Aunque esta conclusión parece acorde con algunos de los datos obtenidos en investigaciones realizadas al respecto (Gómez-Crespo et al., 1995), aún se requiere de un mayor caudal de estudios experimentales que contrasten la validez de dicha hipótesis.

Siguiendo esta línea, en el presente trabajo analizamos el cambio en las concepciones de los alumnos sobre mecánica a través de la enseñanza. Para ello evaluamos el cambio no sólo en respuestas o concepciones puntuales, sino también en su conjunto desde una óptica como la que Monk (1995) propone a través de su idea de estructuración. La hipótesis fundamental que orienta el trabajo consiste en suponer que no sólo encontraremos variaciones en las respuestas puntuales que aportan los alumnos en tareas específicas, sino también cambios profundos que afecten a los criterios que utilizan para responder o, lo que es lo mismo, en los esquemas en que subyacen sus respuestas. Concretamente, pensamos que, junto a cambios positivos observables en las respuestas aisladas de las distintas preguntas, se apreciará también una reestructuración de las teorías implícitas en que subyacen esas respuestas, desde aquéllas que constituyen la lógica intuitiva de sentido común hasta una estructura más coherente y próxima a la ciencia formal.

\section{MÉTODO}

\section{Muestra}

La muestra utilizada constaba de 85 alumnos de $2^{\circ}$ de BUP. Éstos procedían de tres grupos de clase de un mismo centro perteneciente a una capital de provincia, instruidos todos ellos por un mismo profesor. La formación académica previa sobre los tópicos incluidos en los cuestionarios se limitaba a una breve introducción recibida durante $7^{\circ}$ de EGB.

\section{Diseño de la investigación y del proceso instruccional}

El enfoque de la investigación fue de tipo casi experimental, con un diseño pretest-postest sin grupo de control, analizando el cambio conceptual alcanzado.

Desde un punto de vista instruccional, conviene señalar que los materiales utilizados constituían una adaptación de los elaborados por Hierrezuelo y otros (1993). Dichos materiales están estructurados en unidades didácticas en forma de programas-guía de actividades (Furió y Gil, 1980), planteadas bajo un tratamiento continuado de las ideas previas de los alumnos a través de procesos de captura y cambio conceptual, entre otros.

Los temas sobre los que se evaluó el cambio conceptual alcanzado fueron los relacionados con el movimiento y las fuerzas. La enseñanza de estos temas abarcó un período de unas 14 semanas, a razón de 4 sesiones semanales de 50 minutos cada una de duración. El profesor implicado en el proceso de instrucción era el propio autor de esta investigación, el cual tenía ya una experiencia continuada durante varios años en la aplicación de este tipo de metodología didáctica y en el uso de estos materiales.

\section{Instrumentos}

Como instrumentos de recogida de información elaboramos dos cuestionarios, A y B, cada uno integrado por siete preguntas. Las tareas de ambos cuestionarios eran muy similares, si bien cambiaba el contex to involucrado en la situación propuesta. En cada pregunta, los alumnos debían valorar su posición frente a tres ítems o afirmaciones que recogían interpretaciones o predicciones acerca de los fenómenos tratados. En total tenían que evaluar 42 ítems, 21 por cada uno de los cuestionarios.

A la hora de seleccionar los tópicos a estudiar, se ha tenido en cuenta cuáles son los contenidos habituales que se imparten en la enseñanza secundaria y cuáles son las concepciones más estudiadas en la investigaciones sobre enseñanza de las ciencias (Hierrezuelo y Montero, 1988). Los tópicos abordados en las tareas y las concepciones particulares que dentro de cada una se analizaban se muestran en la tabla I.

El formato de presentación de los ítems era tipo Likert con siete niveles que recorrían un arco de plausibilidad 
Tabla I

Temas abordados en las preguntas y concepciones correspondientes.

\begin{tabular}{|c|c|c|c|}
\hline \multirow{2}{*}{$\begin{array}{c}\text { Temas tratados } \\
\text { en los enunciados }\end{array}$} & \multirow{2}{*}{$\begin{array}{c}\text { Concepciones implicadas } \\
\text { en los ítems }\end{array}$} & \multicolumn{2}{|c|}{ Situación planteada } \\
\hline & & Cuestionario A & Cuestionario B \\
\hline $\begin{array}{l}\text { 1. Influencia sobre el } \\
\text { peso de la topología } \\
\text { de un sistema. }\end{array}$ & $\begin{array}{l}\text { C1.1: Los cuerpos pesan más cuando se sitúan sobre el objeto que } \\
\text { les sirve de soporte. } \\
\text { C1.2: Los cuerpos pesan más cuando se sitúan colgados del objeto } \\
\text { que les sirve de soporte. } \\
\text { C1.3: El peso de los cuerpos no varía según la manera cómo se } \\
\text { sitúen.* }\end{array}$ & $\begin{array}{l}\text { Dos bolas unidas me- } \\
\text { diante una cuerda que } \\
\text { se sostiene directamen- } \\
\text { te encima de la mano } \\
\text { o se cuelgan una a cada } \\
\text { lado. }\end{array}$ & $\begin{array}{l}\text { Una bola que se colo- } \\
\text { ca encima de un mue- } \\
\text { lle o se cuelga de él. }\end{array}$ \\
\hline $\begin{array}{l}\text { 2. Influencia del peso } \\
\text { sobre el movimiento } \\
\text { vertical de graves. }\end{array}$ & $\begin{array}{l}\text { C2.1: Los objetos más ligeros son más fácilmente acelerados por } \\
\text { la gravedad. } \\
\text { C2.2: Los objetos pesados son más fácilmente acelerados por la } \\
\text { gravedad. } \\
\text { C2.3: El peso de los cuerpos no afecta a la aceleración de graves.* }\end{array}$ & $\begin{array}{l}\text { Dos bolas que se lan- } \\
\text { zan hacia arriba con la } \\
\text { misma velocidad, una } \\
\text { de más peso que otra. }\end{array}$ & $\begin{array}{l}\text { Dos bolas que se de- } \\
\text { jan caer desde alturas } \\
\text { iguales, una de más } \\
\text { peso que otra. }\end{array}$ \\
\hline $\begin{array}{l}\text { 3. Relación entre at- } \\
\text { mósfera y gravedad- } \\
\text { peso. }\end{array}$ & $\begin{array}{l}\text { C3.1: La ausencia de aire no afecta al peso ni a la gravedad.* } \\
\text { C3.2: Si no hay aire, no hay gravedad ni peso. } \\
\text { C3.3: Si no hay aire, no hay gravedad pero sí peso. }\end{array}$ & $\begin{array}{l}\text { Objeto que se deja caer } \\
\text { en el interior de una } \\
\text { habitación de la que } \\
\text { se ha extraído todo el } \\
\text { aire. }\end{array}$ & $\begin{array}{l}\text { Un objeto se pesa me- } \\
\text { diante una báscula en- } \\
\text { cerrada en un recipiente } \\
\text { del que se ha extraído } \\
\text { todo el aire. }\end{array}$ \\
\hline $\begin{array}{l}\text { 4. Trayectoria de un } \\
\text { objeto que gira y, de } \\
\text { repente, es dejado en } \\
\text { libertad. }\end{array}$ & $\begin{array}{l}\text { C4.1: El objeto tiende a seguir girando. } \\
\text { C4.2: El objeto sale en línea recta en dirección oblicua, intermedia } \\
\text { entre la tangencial y la radial hacia afuera. } \\
\text { C4.3: El objeto sale en línea recta tangente a la trayectoria.* }\end{array}$ & $\begin{array}{l}\text { Una bola se hace girar } \\
\text { atada a una cuerda y } \\
\text { de repente se rompe. }\end{array}$ & $\begin{array}{l}\text { Una bola que sale de } \\
\text { un tubo espiral a tra- } \\
\text { vés del cual ha sido } \\
\text { lanzada. }\end{array}$ \\
\hline $\begin{array}{l}\text { 5. Igualdad o no de } \\
\text { fuerzas en una inte- } \\
\text { racción (choques). }\end{array}$ & $\begin{array}{l}\text { C5.1: Sólo el cuerpo más pesado y que inicia la acción ejerce } \\
\text { fuerza. } \\
\text { C5.2: Ambos objetos se ejercen una fuerza mutua. Pero es el } \\
\text { objeto más pesado y que inicia la acción el que aplica una mayor } \\
\text { fuerza. } \\
\text { C5.3: Las fuerzas que se ejercen dos cuerpos son siempre iguales.* }\end{array}$ & $\begin{array}{l}\text { Un camión a gran ve- } \\
\text { locidad choca contra } \\
\text { una bicicleta estacio- } \\
\text { nada en el andén. }\end{array}$ & $\begin{array}{l}\text { Un niño golpea con el } \\
\text { pie un balón de fútbol. }\end{array}$ \\
\hline $\begin{array}{l}\text { 6. Análisis de fuerzas } \\
\text { en movimientos ascen- } \\
\text { dentes bajo la acción } \\
\text { de la gravedad. }\end{array}$ & $\begin{array}{l}\text { C6.1: Sólo hay una fuerza, que actúa en la dirección y sentido del } \\
\text { movimiento. } \\
\text { C6.2: Sólo hay una fuerza, el peso.* } \\
\text { C6.3: Hay dos fuerzas: el peso y otra en la dirección y sentido del } \\
\text { movimiento. }\end{array}$ & $\begin{array}{l}\text { Una bola que se lanza } \\
\text { verticalmente hacia } \\
\text { arriba. }\end{array}$ & $\begin{array}{l}\text { Un balón de fútbol en } \\
\text { trayectoria ascenden- } \\
\text { te tras ser lanzado en } \\
\text { el saque de una falta. }\end{array}$ \\
\hline $\begin{array}{l}\text { 7. Velocidad en mo- } \\
\text { vimientos de caída li- } \\
\text { bre bajo la acción de } \\
\text { la gravedad. }\end{array}$ & $\begin{array}{l}\text { C7.1: El objeto recorre distancias iguales en tiempos iguales. } \\
\text { C7.2: Cada vez recorre mayor espacio en un tiempo menor.* } \\
\text { C7.3: Cada vez recorre menor espacio en un tiempo mayor. }\end{array}$ & $\begin{array}{l}\text { Un objeto en caída } \\
\text { libre desde cierta } \\
\text { altura. }\end{array}$ & $\begin{array}{l}\text { Una bola que descien- } \\
\text { de por un plano incli- } \\
\text { nado sin rozamiento. }\end{array}$ \\
\hline
\end{tabular}

* Indica la opción teóricamente más adecuada desde el punto de vista científico. 
que iba desde el total acuerdo (puntuación +3 ) hasta el desacuerdo total (puntuación -3). Con este tipo de técnicas queríamos ser fieles al marco teórico utilizado (Oliva, 1999b), dando posibilidad a que se manifestaran los siguientes aspectos:

1) El compromiso de un alumno con una determinada idea suele no ser cuestión de todo o nada, sino de grado, existiendo distintos niveles de acuerdo o plausibilidad.

2) Un mismo alumno podría identificarse con más de una opción, incluso cuando éstas son de carácter contradictorio.

Los ítems propuestos eran transcripciones de respuestas y argumentos aportados por alumnos en un estudio previo realizado empleando cuestionarios de explicación abierta (Oliva, 1994). El orden de presentación de las tareas era idéntico para ambos cuestionarios, si bien dentro de cada tarea los tres ítems estaban ordenados de un modo diferente. No obstante, a efectos de la exposición hemos situado los ítems en el mismo orden en ambos casos para facilitar así la lectura e interpretación de los datos. El apéndice recoge un ejemplo de tarea planteada en uno de los cuestionarios.

Las pruebas se administraron como pretest a comienzos de curso, antes de empezar los temas de mecánica, y más tarde como postest a finales de curso, dos meses después de acabar las unidades didácticas referentes a las concepciones abordadas en los cuestionarios.

Los cuestionarios se presentaron en horas habituales de clase. Aunque no se puso un límite de tiempo para su realización, todos lo completaron en menos de treinta minutos. Los estudiantes sabían que participaban en un sondeo de sus concepciones y que el resultado no tendría incidencia en sus calificaciones.

\section{Análisis de datos}

Los datos de las pruebas empleadas fueron organizados en una matriz de datos e introducidos en una base de datos de un ordenador. El tratamiento estadístico se efectuó mediante el paquete SPSS, aplicando análisis descriptivo, estudios de correlación, análisis factorial y de fiabilidad, y análisis de diferencias mediante la $t$-student para muestras dependientes.

\section{RESULTADOS}

\section{Análisis descriptivo de los datos}

Se efectuó un primer análisis de resultados comparando los datos obtenidos en los distintos ítems del pretest y del postest. Dicho análisis se muestra en las figuras 1 y 2 . Un rápido repaso a esos gráficos permite comprobar los siguientes aspectos:
1)En más de la mitad de las tareas del pretest, los ítems que representaban opciones acordes con el punto de vista científico arrojaron promedios de puntuaciones negativas. Este hecho indica un bajo grado de aceptación inicial de estas opciones. Las excepciones las encontramos en las tareas 1 y 7 del cuestionario A, y en las tareas 3, 4 y 7 del cuestionario B. En ellas los promedios sí adoptaron valores positivos, mostrando así un cierto grado de plausibilidad para la muestra en su conjunto.

2) También en el pretest, ciertas ideas habituales en los estudios sobre concepciones alternativas alcanzaron un nivel relativamente alto de plausibilidad. Tal es el caso, por ejemplo, de la conexión que se establece entre el peso de un objeto y la rapidez del movimiento vertical de graves (ítem C2.2), la desigualdad de fuerzas en una interacción (ítem C5.2) o la asociación entre fuerza y movimiento (ítem C6.3).

3)En el postest, se aprecia, por lo general, en términos comparativos, un ascenso en la plausibilidad de las ideas acordes con el punto de vista científico y un descenso en la de las ideas alternativas. En algunos casos, no obstante, las variaciones fueron escasas, lo que sugiere que el cambio conceptual en determinadas nociones ha sido limitado.

Los datos comentados sugieren un cierto nivel de cambio en algunas de las concepciones preinstruccionales. Sin embargo proporcionan una imagen poco estructurada del alumno, que no tiene en cuenta cómo se organiza el conocimiento ni los cambios que se verifican en esa organización. Además, desde un punto de vista estadístico, los resultados que aporta cada ítem por separado son de una dudosa fiabilidad, lo que resta significatividad a las conclusiones que a partir de ellos podemos extraer. Todo ello no hace sino aconsejar otras formas de analizar las concepciones y otras maneras de evaluar los cambios que se producen en las mismas. A ello dedicaremos espacio a continuación estudiando cuáles son los esquemas subyacentes a las respuestas concretas.

\section{Construcción de escalas y cambios que se observan en las mismas}

Con objeto de proporcionar una visión más estructurada del conocimiento intuitivo del alumno, se ha construido una serie de escalas para el pretest combinando los resultados de ítems de contenido análogo. El uso posterior de estas escalas permitió contar con datos más fiables que los que se desprenden de cada ítem por separado (Licht y Thijs, 1990). El método empleado ha sido ya descrito en un trabajo anterior (Oliva, 1999a) y pasamos a reseñarlo a continuación, brevemente.

En un primer paso se llevó a cabo una selección de los ítems más representativos de acuerdo con los siguientes criterios:

1) Todos los ítems que incluían concepciones acordes con el punto de vista científico fueron incluidos. 
Figura 1

Comparación de resultados del pretest y del postest para las concepciones acordes con el punto de vista científico.

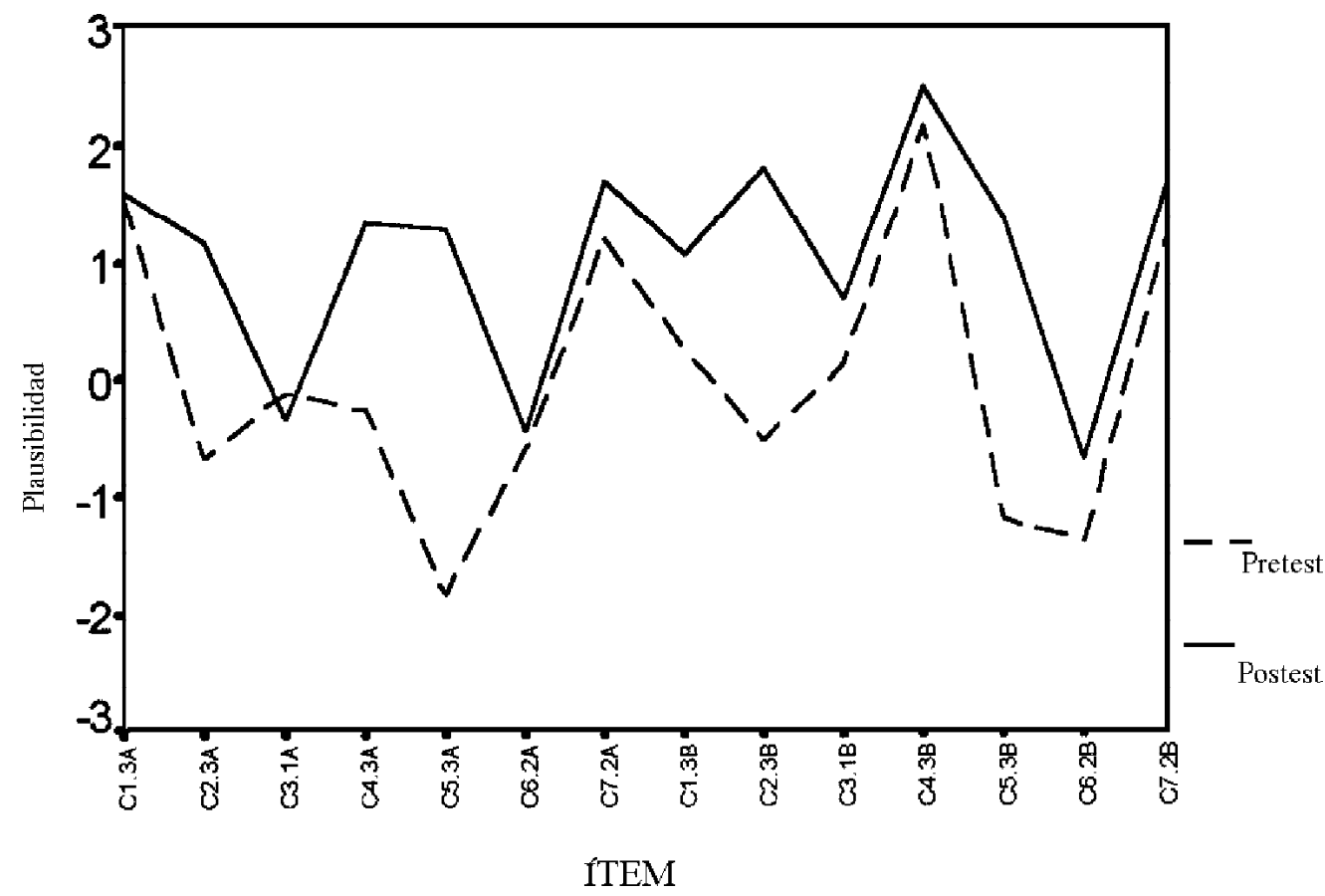

Figura 2

Comparación de resultados del pretest y del postest para las concepciones alternativas.

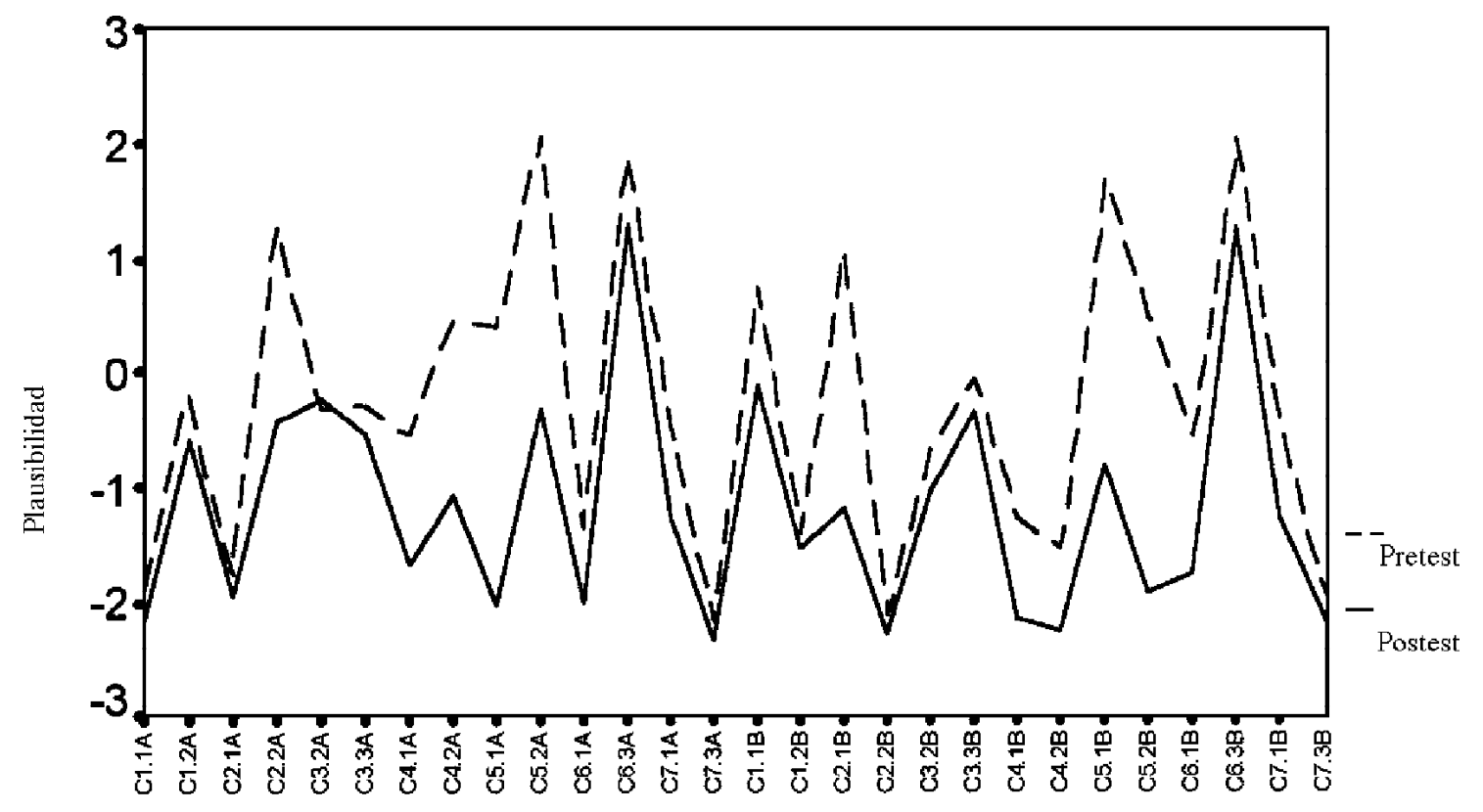

ITEM 
Tabla II

Interpretación y fiabilidad de las escalas del pretest y del postest.

\begin{tabular}{|c|c|c|c|c|c|}
\hline Escala & Polo (+) & Polo (-) & $\begin{array}{l}\text { Número } \\
\text { de ítems }\end{array}$ & \multicolumn{2}{|c|}{$\begin{array}{c}\alpha \text { de Cronbach } \\
\text { Pretest } \quad \text { Postest }\end{array}$} \\
\hline $\mathrm{E}$ & $\begin{array}{l}\text { Conservación del peso ante variaciones } \\
\text { en la configuraciónde un sistema. } \\
\text { C1.3(A) C1.3(B) }\end{array}$ & $\begin{array}{l}\text { Los cuerpos pesan más cuando } \\
\text { se cuelgan. } \\
\text { C1.2(A) C1.2(B) }\end{array}$ & 4 & 0,64 & 0,82 \\
\hline E2 & $\begin{array}{l}\text { Independencia del movimiento de } \\
\text { graves respecto al peso del objeto. } \\
\text { C2.3(A) C2.3(B) }\end{array}$ & $\begin{array}{l}\text { Los cuerpos más pesados se ven } \\
\text { más afectados por la gravedad. } \\
\text { C2.2(A) C } 2.2(\mathrm{~B})\end{array}$ & 4 & 0,74 & 0,89 \\
\hline E3 & $\begin{array}{l}\text { Independencia del peso de la } \\
\text { presencia o no de aire. } \\
\text { C } 3.1(\text { A) C } 3.1 \text { (B) }\end{array}$ & $\begin{array}{l}\text { Los cuerpos no caen o no pesan } \\
\text { en ausencia de aire. } \\
\text { C3.2(A) C3.2(B) }\end{array}$ & 4 & 0,70 & 0,69 \\
\hline $\mathrm{E} 4$ & $\begin{array}{l}\text { Inercia rectilínea tangente a la } \\
\text { trayectoria. } \mathrm{C} 4.3(\mathrm{~A}) \mathrm{C} 4.3(\mathrm{~B})\end{array}$ & $\begin{array}{l}\text { Inercia circular. Los cuerpos que } \\
\text { giran siguen girando al quedar en } \\
\text { libertad. C4.1(A) C4.2(B) }\end{array}$ & 4 & 0,57 & 0,62 \\
\hline E5A & $\begin{array}{l}\text { Igualdad de fuerzas en un choque. } \\
\text { C5.3(A) C } 5.3(\mathrm{~B})\end{array}$ & $\begin{array}{l}\text { El objeto de mayor masa y mayor } \\
\text { velocidad ejerce una fuerza mayor } \\
\text { en un choque. C5.2(A) C } 5.2(B)\end{array}$ & 4 & 0,74 & 0,91 \\
\hline E5B & $\begin{array}{l}\text { En un choque los dos cuerpos } \\
\text { se ejercen fuerza mutua. }\end{array}$ & $\begin{array}{l}\text { Sólo el objeto de mayor masa y } \\
\text { mayor velocidad ejerce fuerza en } \\
\text { un choque. C5.1(A) C } 5.1(\mathrm{~B})\end{array}$ & 2 & 0,62 & 0,76 \\
\hline E6 & $\begin{array}{l}\text { Sobre un cuerpo que asciende } \\
\text { actúan varias fuerzas. } \\
\text { C6.3(A) C6.3(B) }\end{array}$ & $\begin{array}{l}\text { Sobre un cuerpo que asciende } \\
\text { actúa sólo una fuerza. } \\
\text { C6.1(A) C6.1(B) C6.2(A) C6.2(B) }\end{array}$ & 6 & 0,69 & - \\
\hline E7 & $\begin{array}{l}\text { El movimiento de caída es acelerado. } \\
\text { C7.2(A) C7.2(B) }\end{array}$ & $\begin{array}{l}\text { El movimiento de caída es } \\
\text { a velocidad constante. } \\
\text { C7.1(A) C7.1(B) }\end{array}$ & 4 & 0,82 & 0,84 \\
\hline $\mathrm{E} 8 \mathrm{a}$ & & $\begin{array}{l}\text { Asociación fuerza-movimiento (I): } \\
\text { sobre un cuerpo que asciende actúa } \\
\text { sólo una fuerza, orientada en el } \\
\text { sentido del movimiento. } \\
\text { C6.1(A) C6.1(B) }\end{array}$ & 2 & 0,60 & 0,80 \\
\hline $\mathrm{E} 8 \mathrm{~b}$ & $\begin{array}{l}\text { Noción newtoniana de fuerza: } \\
\text { sobre un cuerpo que asciende actúa } \\
\text { sólo la fuerza peso. } \\
\text { C6.2(A) C6.2(B) }\end{array}$ & $\begin{array}{l}\text { Asociación fuerza-movimiento (II): } \\
\text { sobre un cuerpo que asciende actúa } \\
\text { además del peso una fuerza en el } \\
\text { sentido del movimiento. } \\
\text { C6.3(A) C6.3(B) }\end{array}$ & 4 & - & 0,91 \\
\hline
\end{tabular}

Entre paréntesis se indica A o B para referirse al cuestionario del que se había extraído el ítem.

2) Se incluyeron también aquellos ítems que recogían respuestas acordes con concepciones alternativas de una cierta plausibilidad (-1).

3) No obstante, dentro de estos últimos, se descartaron todos aquellos ítems que no mostraron un nivel significativo de correlación con sus equivalentes en la otra prueba.
Como resultado de dicho proceso de selección se conservaron sólo 32 de los 42 ítems incluidos en las pruebas. Estos ítems se dividieron en siete grupos de acuerdo con su afinidad temática, uno por cada una de las siete tareas involucradas en los cuestionarios. La validez interna de cada uno de los agrupamientos se evaluó, respectivamente, a través de una primera tanda de análisis de componentes principales. En primera instancia sólo se 
consideró un factor como solución de cada análisis. El nivel de ajuste al modelo fue bueno en todos los casos, a excepción hecha del grupo de ítems correspondientes a las tareas número 5 de los cuestionarios. En este caso, fue preciso introducir un segundo factor para obtener un nivel de ajuste aceptable. Como resultado de todo este proceso fue posible resumir la información disponible en sólo ocho escalas (escalas 1, 2, 3, 4, 5A, 5B, 6 y 7), con cada ítem contribuyendo a una y sólo a una de las escalas.

Las escalas solían tener una naturaleza bipolar, ya que incluían concepciones acordes con el punto de vista científico y también concepciones alternativas. Cada escala se obtuvo mediante adición directa de las puntuaciones de los correspondientes ítems, verificándose luego una recodificación de los valores obtenidos para que encajasen en un rango comprendido en el intervalo $[+1,-1]$, que es más manejable y fácil de interpretar. La asignación de los polos positivo y negativo de cada escala se realizó utilizando como criterio la proximidad del extremo positivo al punto de vista científico, lo cual demostró que evitaba trabajar con correlaciones negativas entre variables. La composición de cada escala, su fiabilidad y otros datos de interés se recogen en la tabla II.

Con posterioridad definimos una novena escala, E8A, cuyos ítems estaban ya incluidos en la escala 6. Se procedió de esta forma sólo a efectos comparativos con las escalas delimitadas en el postest, las cuales analizaremos más tarde.

En cuanto al significado de cada una de las ocho escalas del pretest, siete de ellas fueron relativamente fáciles de interpretar identificándose un extremo coherente con el punto de vista científico y otro que recogía una visión alternativa (escalas 1, 2, 3, 4, 5A, 5B y 7).

La escala restante (escala 6) resultó la más compleja y problemática. Si bien, en principio, se esperaba que ayudaría a evaluar la posición del alumno entre la visión newtoniana del concepto de fuerza (C6.2) frente a la asociación fuerza-movimiento de las posturas aristotélico-escolásticas (C6.1 y C6.3), los datos obtenidos nos obligaron a replantear el significado de la misma. De forma sorprendente los ítems C6.1 y C6.2 de ambos cuestionarios correlacionaban positivamente entre sí, integrándose en un mismo extremo de la escala en el lado opuesto en el que se situaban los ítems C6.3. Por ello, en su interpretación final, se consideró que esta dimensión marcaría la tendencia a abordar el problema de una forma analítica, contemplando la posibilidad de actuación de varias fuerzas a la vez, frente a la tendencia a hacerlo a partir de una sola. El primero de estos extremos fue el que mejor correlacionó con el polo positivo de las otras escalas y por ello se le asignó el polo positivo.

Mediante un proceso semejante logramos resumir los datos del postest en una serie de nueve escalas (escalas, 1, 2, 3, 4, 5A, 5B, 7, 8A y 8B). La interpretación de las mismas y sus valores de fiabilidad se recogen también en la tabla II.
Antes de pasar al estudio cuantitativo de las transformaciones que han operado sobre las concepciones de los alumnos, conviene realizar un balance cualitativo de los cambios ocurridos. Ello puede hacerse a partir de la comparación del significado de las escalas definidas en uno y otro caso. Sobre ello hay que señalar que siete de las ocho escalas iniciales conservaron su validez interna en el postest, lo que denota un importante grado de continuidad en los marcos referenciales que orientaban las respuestas de los alumnos. No obstante, la escala 6 sufrió un proceso de reestructuración a través de la enseñanza, desdoblándose en dos escalas diferentes, E8A y E8B respectivamente. La primera de ellas se definió de un modo unipolar y para ello se tomó como referente el significado del extremo negativo del continuo. Correspondía a un efecto residual de la escala E6 del pretest, si bien aquí prevalecía la idea de impetus como única causa que acciona el movimiento del objeto y ni siquiera se contemplaba el peso del cuerpo como posibilidad. La segunda, en cambio, parecía claramente de una naturaleza bipolar. Un extremo incluía sólo la fuerza peso, que fue identificado como el extremo adecuado desde el punto de vista científico, y otro que, además del peso, incluía una fuerza en la dirección y sentido del movimiento. De ahí se deduce que la dicotomía entre el estudio dinámico de un sistema a partir de una sola causa o la posibilidad de varias causas a la vez daba paso después de la instrucción a una visión en la que la idea de fuerza evolucionaba notablemente, si bien la asociación entre fuerza y movimiento, como veremos a continuación, no llegó a ser superada al menos dentro de los límites de lo que hubiéramos deseado.

En cuanto a los valores de fiabilidad de las escalas, se constata, por lo general, un cierto aumento del coeficiente de consistencia interna a través de la instrucción.

En la tabla III se indican los promedios de valores alcanzados en las escalas del pretest y del postest. Además, dicha tabla recoge los valores de cambio observados en cada una de las escalas y el grado de significatividad estadística de dicho cambio, que se desprende de la prueba de comparación de datos para medidas dependientes. El hecho de que las distribuciones de puntuaciones en las distintas escalas se aproximara bastante a la curva normal nos animó a emplear una estadística de tipo paramétrico en nuestras inferencias.

Desde un punto de vista del cambio que se ha producido en cada escala a través de la enseñanza, puede decirse que éste ha sido positivo y bastante notorio en las escalas relacionadas con la influencia de la masa en el movimiento de graves (escala E2) y la idea de fuerza como interacción (escalas E5A y E5B). También puede considerarse aceptable en las escalas sobre la noción de inercia (escala E4) y el estudio dinámico de un sistema de la escala E8A. En el resto de escalas, el cambio resulta discreto. Así, por ejemplo, resulta bastante limitado en las concepciones correspondientes a la influencia sobre el peso de la configuración de un sistema (escala E1) y el movimiento descrito por un objeto bajo la acción de la gravedad (escala E7). Por otra parte, ni siquiera llega a 
Tabla III

Evolución de las concepciones de los alumnos a través de las diferentes escalas y prueba estadística para las diferencias pretest-postest.

\begin{tabular}{|c|c|c|c|c|c|c|c|}
\hline \multirow[t]{2}{*}{ Escala } & \multicolumn{2}{|c|}{ Pretest } & \multicolumn{2}{|c|}{ Postest } & \multicolumn{2}{|c|}{ Cambio } & \multirow{2}{*}{$\begin{array}{c}\text { Prueba t } \\
\text { Student }\end{array}$} \\
\hline & Media & DS & Media & DS & Media & DS & \\
\hline E1 & 0,10 & 0,47 & 0,28 & 0,52 & $+0,18$ & 0,50 & $* *$ \\
\hline E2 & $-0,30$ & 0,55 & 0,38 & 0,60 & $+0,67$ & 0,69 & $* * *$ \\
\hline E3 & 0,08 & 0,50 & 0,14 & 0,48 & $+0,05$ & 0,46 & NS \\
\hline $\mathrm{E} 4$ & 0,31 & 0,39 & 0,63 & 0,39 & $+0,32$ & 0,46 & $* * *$ \\
\hline E5A & $-0,57$ & 0,35 & 0,31 & 0,63 & $+0,88$ & 0,70 & $* * *$ \\
\hline E5B & $-0,15$ & 0,59 & 0,66 & 0,42 & $+0,80$ & 0,66 & $* * *$ \\
\hline E6 & 0,44 & 0,35 & - & - & - & - & - \\
\hline E7 & 0,28 & 0,55 & 0,49 & 0,48 & $+0,20$ & 0,51 & $* *$ \\
\hline E8A & 0,32 & 0,50 & 0,63 & 0,43 & $+0,30$ & 0,54 & $* * *$ \\
\hline E8B & - & - & $-0,31$ & 0,65 & - & - & - \\
\hline
\end{tabular}

$* * * \mathrm{P}<0,001 ; * * \mathrm{p}<0,01 ; * \mathrm{p}<0,05 ; \mathrm{NS}=$ No significativo

superar el umbral de significatividad estadística en el caso de la influencia del aire en el peso de un objeto (escala E3).

En cuanto a la escala E8B, se aprecia que la puntuación final se mueve en la zona negativa del continuo. Ello denota un alto nivel de aceptación de la idea de asociación entre fuerza y movimiento y sugiere una insuficiente repercusión de la instrucción en esta dirección. Esta noción parece diferenciarse de otras ideas después de la enseñanza formal, cobrando un estatus propio independiente de otras ideas, pero no logra desaparecer ni ser cambiada por el punto de vista newtoniano del movimiento.

Aunque el estudio de los resultados que proporcionan las escalas puede ser más interesante que el que aportan los diversos ítems por separado, pensamos que todavía podría accederse a un análisis más profundo de los datos investigando las relaciones existentes entre los esquemas conceptuales evaluados mediante las escalas. A ello dedicamos espacio en el siguiente apartado.

\section{Relaciones entre escalas y cambios detectados}

El estudio de las relaciones existentes entre las diversas escalas se llevó a cabo mediante el coeficiente de correlación de Pearson. En el pretest no hemos incluido la escala E8a, dado que los ítems que la integraban ya estaban incluidos en la escala E6. Con dicha exclusión pretendíamos evitar introducir información redundante en las correlaciones.

Las correlaciones obtenidas en el pretest fueron semejantes a las expuestas en un estudio anterior (Oliva, 1999a), si bien aquí los valores fueron algo mayores. En esta ocasión tuvieron en 0,48 su valor máximo (escalas E5B y E6) y alcanzaron los límites de significatividad estadística en uno de cada cinco casos. En el postest, las correlaciones resultaron algo superiores en términos generales, teniendo como valor máximo 0,52 (escalas E5B y E8A) y siendo estadísticamente significativas en casi la mitad de las ocasiones. Estos valores más altos de correlación entre escalas concuerdan bien con el aumento también detectado en la fiabilidad de las escalas (Tabla III) y denotan un incremento de la coherencia interna de las respuestas de los alumnos.

Al lado de estos cambios en las correlaciones, se apreciaron además algunos cambios cualitativos importantes en la gama de valores obtenidos, lo cual nos animó a estudiar los cambios ocurridos en la organización de los mismos. Con objeto de acceder a dicha organización se efectuó una segunda ronda de análisis de componentes principales, esta vez utilizando las escalas del pretest, por un lado, y del postest, por otro, como variables incluidas en los respectivos análisis.

El análisis arrojó en el pretest una solución con cuatro factores que explicaban el $71 \%$ de la varianza total. La tabla IV recoge las cargas factoriales. Los factores obtenidos coinciden con los mismos que obtuvimos e interpretamos en un trabajo anterior ayudados, entre otras cosas, por el uso de entrevistas (Oliva, 1999a). El significado de cada uno podría también identificarse con el de los factores allí discutidos:

- Factor 1: Varias fuerzas o una sola fuerza en el estudio del comportamiento dinámico de un sistema (escalas E5B y E6), según que los alumnos postulasen la presencia de una o más fuerzas en los análisis efectuados. 
- Factor 2: Conservación o no conservación del peso ante ciertas modificaciones (escalas E1 y E3).

- Factor 3: Visión lógico-analítica o visión intuitiva en el estudio del tipo de movimiento de un objeto (escalas E4 y E7), según que los razonamientos tengan en cuenta o no los cambios que se verifican en las condiciones del fenómeno a medida que se produce.

- Factor 4: Diferenciación o identificación de la magnitud de una causa con la de sus efectos (escalas E2 y E5A), según que se asocie o no la magnitud de una fuerza con la del efecto que ocasiona.

Tabla IV

Análisis de componentes principales para las escalas de mecánica en el pretest.

\begin{tabular}{|l|c|c|c|c|}
\hline \multirow{2}{*}{ Variables } & \multicolumn{4}{|c|}{ Rotación VARIMAX } \\
\hline & F1 & F2 & F3 & F4 \\
\hline E5B & 0,87 & - & - & - \\
\hline E6 & 0,83 & - & - & - \\
\hline E1 & - & 0,79 & - & - \\
\hline E3 & - & 0,73 & - & - \\
\hline E7 & - & - & 0,88 & - \\
\hline E4 & - & - & 0,75 & - \\
\hline E2 & - & - & - & 0,84 \\
\hline E5A & - & - & - & 0,74 \\
\hline
\end{tabular}

Sólo se recogen los valores de cargas factoriales superiores a 0,40 .

Como ya mencionamos en el trabajo citado, estos factores concuerdan bien con algunas de las reglas causales empleadas en estudios teóricos y de corte cualitativo para explicar ciertas semejanzas observadas en las concepciones a lo largo de distintos tópicos. De forma más específica, los factores 1, 2 y 4 parecen coherentes con dos de las estructuras de pensamiento postuladas por Pozo y otros (Pozo et al., 1991; Pozo y Gómez-Crespo, 1997) para distinguir las características del conocimiento implícito de sentido común y las del conocimiento científico. Concretamente, el primero y el cuarto se alinean con la dimensión referente a la interacción versus causalidal lineal simple:

«Los alumnos tienden a recurrir a un esquema causal muy simple para explicar los acontecimientos según el cual la relación entre causa y efecto es lineal y en un solo sentido. Sin embargo, la mayor parte de las teorías científicas requieren entender las situaciones como una interacción de sistemas en las que como mínimo se produce una de las dos situaciones siguientes: a) la relación causa-efecto no es en un solo sentido, sino que implica una relación recíproca $[. .$.$] ; b) la relación implica$ no sólo una causa sino la interacción entre varias causas que se coordinan para producir un efecto dado» (Pozo y Gómez-Crespo, 1997, p. 96).
Mientras tanto, el segundo factor se relaciona con el esquema de conservación versus no-conservación, que aquí se manifiesta a través de la conservación o no del peso ante una alteración de alguna de las variables del fenómeno.

Finalmente, la tercera dimensión analizada también parece tener un componente general importante y formaría parte de una tendencia causal más amplia al estilo de la propuesta por autores como Acevedo (1990) o Touger y otros (1995). Estos autores han encontrado que algunas de las explicaciones de los alumnos en tareas de ciencias se basan en impresiones personales superficiales que no entran en la lógica del fenómeno, en contraposición a otras en las que se razona de un modo estructurado mediante inferencias causales que identifican los conceptos relevantes de la tarea y las diferencias que existen entre los estados iniciales y finales del fenómeno. Esta dimensión manifestaría, pues, la tendencia de los alumnos a abordar los problemas de movimiento de un modo lógico utilizando argumentos causales más o menos articulados, frente a la tendencia a hacerlo de un modo intuitivo sin un control de las variables que participan en el fenómeno. En esta ocasión, la variable a tener en cuenta en un caso sería la acción central que mantiene a los cuerpos girando y, en el otro, la variación de velocidad que altera los tiempos empleados en recorrer longitudes iguales.

En el postest, el análisis de componentes principales ponía de manifiesto la existencia de sólo tres factores que explicaban el $60 \%$ de la varianza (Tabla V). La adición de un cuarto factor hizo aumentar la varianza explicada hasta un $70 \%$, pero no fue tenido en cuenta, ya que arrojaba un valor propio inferior a la unidad $(0,87)$

Tabla V

Análisis de componentes principales para las escalas de mecánica en el postest.

\begin{tabular}{|l|c|c|c|}
\hline Variables & \multicolumn{3}{|c|}{ Rotación VARIMAX } \\
\hline & F1 & F2 & F3 \\
\hline E5B & 0,79 & - & - \\
\hline E5A & 0,77 & - & - \\
\hline E8A & 0,71 & - & - \\
\hline E1 & 0,54 & 0,53 & - \\
\hline E3 & - & 0,79 & - \\
\hline E7 & - & 0,75 & - \\
\hline E4 & - & - & 0,76 \\
\hline E2 & - & - & 0,71 \\
\hline E8b & - & 0,43 & 0,47 \\
\hline
\end{tabular}

Sólo se recogen los valores de cargas factoriales superiores a 0,40 .

De la comparación de los análisis del pretest y del postest se infiere un cambio en los factores en los que se ubica 
cada escala, lo cual origina asimismo un cambio en la interpretación de cada uno de ellos. De hecho, el aumento observado en la coherencia entre escalas se traduce aquí en un incremento del número de variables que carga sobre cada factor, lo que sugiere un crecimiento de la complejidad de cada uno y también un aumento de la dificultad de su interpretación. El acercamiento de las concepciones de los alumnos a las nociones científicas que se instruyen parece conllevar asimismo una aproximación entre las distintas concepciones.

El factor 1 constituye una dimensión relacionada con la noción de fuerza como interacción frente al esquema de fuerza como propiedad. Apunta, pues, en la misma dirección que el tercer principio de la dinámica, frente a una visión más simplista de la fuerza como propiedad que puede ser transmitida de un cuerpo a otro.

El factor 2 resulta algo más problemático. Su significado no es fácil de descifrar, dado que aparece como una dimensión compleja que engloba a dos subfactores. Uno de ellos guarda ciertas reminiscencias del factor 2 del pretest, en el que se encuadraban las escalas E1 y E3 y que hacía referencia a la conservación o no del peso. Mientras tanto, el otro refleja una dicotomía entre la fuerza como causa de la aceleración o como causa de velocidad, y engloba las escalas E7 y E8b. La existencia de estos dos subfactores pudo comprobarse mediante un análisis factorial posterior contemplando sólo estas cuatro variables, aunque, para no cansar al lector con un baile de cifras, omitiremos dicho análisis de nuestra exposición. En conjunto, estas cuatro escalas quedaban relacionadas bajo una misma dimensión que marcaría tendencias opuestas en la manera de entender el papel de las fuerzas, en general, y de la fuerza-peso, en particular. De un lado, el esquema de fuerza-peso como agente causal que produce aceleración y que es independiente de otros factores y, de otro, la fuerza-peso como un evento que depende de variables externas y que produce un movimiento constante.

Por último, el factor 3 presenta una naturaleza aún más compleja, comportándose como un factor residual que engloba tres escalas cuya relación no acabamos del todo de descifrar. Aunque sólo sea a modo de aventurar una hipótesis, es posible que la asociación detectada pudiera explicarse mediante una dicotomía entre la noción de inercia o la de impetus a la hora de explicar el movimiento de los objetos. Bajo este planteamiento encajaría plenamente la escala 4, como variable que indica la visión de los alumnos acerca de qué le ocurre a un objeto cuando no queda sometido a ninguna fuerza. Los planteamientos de los alumnos aquí se mueven entre la utilización del principio de inercia o la idea de fuerza como algo interno que queda impreso en los objetos y dirige su movimiento (Carrascosa y Gil, 1992). También podría encajar la escala 2 , dado que la asimilación correcta de la independencia del movimiento de graves de la masa de los cuerpos debería pasar por la consideración conjunta de la idea de inercia junto a la de masa gravitatoria. Frente a ello, los alumnos que se dejen guiar por la idea de impetus tenderán a establecer una relación directa entre fuerza y movi- miento, pensando que los cuerpos más pesados caen a mayor velocidad, dado que son impulsados por una fuerza también mayor. Finalmente, esta interpretación podría explicar también la aparición de una carga importante sobre este factor de la escala E8b, en la que entraba en juego la idea de asociación o no entre fuerza y movimiento.

En resumidas cuentas, los tres factores aludidos podrían identificarse de la siguiente forma:

Factor 1: La fuerza como interacción o la fuerza como propiedad (escalas E5A, E5B, E8Ay E1).

Factor 2: La fuerza como causa o la fuerza como evento (escalas E1, E3, E7 y E8b).

Factor 3: El movimiento como inercia o el movimiento como impetus (escalas E2, E4 y E8b).

Si estas interpretaciones son correctas, los polos positivos correspondientes a las tres dimensiones del postest estarían muy cercanos a las tres leyes de la dinámica, correspondiendo el primer factor a la tercera ley (ley de acción-reacción), el segundo factor aproximadamente a la segunda (la fuerza como causa de aceleraciones) y el tercer factor posiblemente a la primera (principio de inercia).

Por encima de las interpretaciones comentadas, las cuales evidentemente reclaman una mayor investigación en este terreno, podemos establecer algunas diferencias importantes en la estructura de relaciones entre escalas del pretest y del postest:

1)Los valores de correlación entre diferentes escalas aumentan notablemente después de la instrucción.

2)Los factores que explican las relaciones entre escalas del pretest corresponden a criterios diferentes a los del pretest.

3)La complejidad de interpretación de los factores aumenta en el postest. Se articulan escalas que en principio no guardaban una relación inmediata. Más que esquemas simples parece que se transforman en esquemas complejos que coordinan diversos subesquemas.

4)En algunas ocasiones del postest, una misma escala parece depender de más de un factor a la vez, lo que sugiere un aumento de complejidad en las decisiones de los alumnos.

5) Como resultado de la enseñanza, se aprecia un notable acercamiento de los criterios que organizan las respuestas de los alumnos al contenido de las leyes de la mecánica.

Todo ello indica un cambio importante en los criterios estructurales que orientan las ideas de los alumnos y también una mayor capacidad para resolver tareas utilizando diversos criterios a la vez. 


\section{CONCLUSIONES}

A lo largo del trabajo hemos ido evaluando los cambios que se producen en las concepciones de los alumnos siguiendo distintos niveles de análisis. Empezando por el más simple de ellos, hemos comparado las respuestas que aportan en ítems individuales, antes y después de la enseñanza. De la comparación efectuada se infiere un cierto cambio en las respuestas, verificándose un relativo aumento de la plausibilidad de algunas de las nociones que resultan apropiadas desde el punto de vista científico y un descenso de algunas ideas alternativas.

En un segundo nivel hemos agrupado las respuestas por escalas según su afinidad temática, que fueron posteriormente validadas mediante un análisis de componentes principales. De la comparación de las escalas resultantes del pretest y del postest se desprende un cierto grado de continuidad en la naturaleza y el significado de la mayor parte de ellas. Se detecta, no obstante, un cambio o reestructuración en una de las escalas construidas que se veía desdoblada en dos después de la enseñanza. Los resultados de las escalas comunes al pretest y al postest muestran un cambio cuantitativo en algunas de ellas, lo que apunta hacia una creciente aproximación de los esquemas de los alumnos hacia el punto de vista científico. En otros casos, los cambios son discretos y, puntualmente, en alguno de ellos no se logra alcanzar siquiera la significación estadística.

Finalmente, en un tercer nivel hemos analizado la estructura factorial de las escalas construidas con objeto de perfilar criterios comunes entre distintas concepciones y esquemas. Como resultado de dicho análisis se observa un aumento notable en el grado de interrelación de las escalas, lo cual se refleja en un aumento de las correlaciones entre ellas. Junto a este cambio se detecta también una variación en el significado de los criterios o factores aislados, un aumento en la complejidad interna de cada factor y una mayor complejidad de las decisiones de los alumnos. Éstas pasarían, de estar dirigidas por una sola dimensión, a apoyarse en ocasiones sobre más de un criterio o factor a la vez.

\section{IMPLICACIONESEDUCATIVAS Y SUGEREN- CIAS PARA INVESTIGACIONES FUTURAS}

Los resultados de la investigación sugieren dos aspectos importantes a tener en cuenta en las investigaciones futuras sobre concepciones de los alumnos. En primer lugar, nos muestran la existencia de un cierto grado de estructuración en las contestaciones de los estudiantes, tanto antes como después de la enseñanza. Dicha organización, lejos de ser simple, podría configurar un entramado jerárquico en el que unas estructuras encajan dentro de otras según un orden que va de mayor a menor generalidad.

En segundo lugar, indican que los cambios que tienen lugar en el conocimiento ocurren no solamente a niveles observables de las respuestas de los estudiantes en pre- guntas o nociones puntuales, sino también en las estructuras de más alto nivel en las cuales subyacen parcialmente las respuestas. Estos resultados son acordes con las ideas de Vosniadou (1994), quien distingue entre teorías específicas y teorías estructurales como dos niveles posibles a tener en cuenta en el pensamiento intuitivo del alumno. También concuerda con el marco teórico que aportan otros trabajos en los que se sugiere la necesidad de tener en cuenta tanto los esquemas generales del alumno como los específicos a la hora comprender el origen de las concepciones alternativas (Marín, 1994; Benarroch y Marín, 1998). Así mismo, parece consistente con propuestas como las de Monk (1995) o Pozo y Gómez-Crespo (1998), quienes postulan diversos niveles de organización estructural para el pensamiento intuitivo de los estudiantes.

Así, pues, el cambio en el conocimiento parece comportar una transformación a varios niveles. Algunos de ellos implican un cambio a gran escala en los esquemas causales del individuo, mientras que otros operan de un modo más fino a través de un cambio en los contenidos específicos en los cuales se concretan esas estructuras. Esto demanda un mayor esfuerzo por parte del profesor, que debería también prestar atención a estos esquemas más generales que aparecen detrás de muchas de las respuestas encontradas en cuestiones concretas. De lo anterior se desprende la necesidad de una cuidadosa revisión de los modelos actuales sobre el cambio conceptual, así como de las estrategias de enseñanza que a partir de los mismos se derivan.

Desde este punto de vista, deberían tenerse en cuenta las limitaciones de las investigaciones y metodologías de enseñanza preocupadas por originar el cambio conceptual a un solo nivel. Por ejemplo, el enfoque de las operaciones formales se ha centrado sólo en el desarrollo de las destrezas lógicas en abstracto sin tener en cuenta la importancia de los contenidos y del contexto en el que éstos se materializan. Los programas de investigación destinados a acelerar el proceso de maduración del alumno han solido fracasar porque no tenían en cuenta la importancia del contexto y del contenido como elementos esenciales que influyen en la adquisición de destrezas intelectuales. Mientras tanto, el movimiento de las concepciones alternativas coloca el acento solamente en los cambios que operan en los niveles más bajos de la jerarquía. Olvida, en consecuencia, los niveles superiores y entiende el cambio como una sustitución de una idea por otra para lo que sería suficiente someter a los alumnos a estrategias de conflicto conceptual en tareas puntuales y específicas. Ello suele provocar que el cambio, cuando se verifica, ocurra parcialmente y de un modo temporal afectando sólo a los elementos superficiales de la estructura.

Se desprende de todo ello la necesidad de integrar ambos enfoques y abordar el problema del cambio conceptual teniendo en cuenta diversos niveles de estructuración. Se debe resaltar, en este sentido, la necesidad de recuperar la idea de reestructuración frente a la de sustitución o desplazamiento, noción esta última que mantiene connotaciones no del todo acordes con la idea de aprendizaje 
como construcción. La enseñanza debería ir dirigida a la evolución de las ideas no a su sustitución. El conflicto conceptual, como fuente de desequilibrios debidos a contradicciones ente predicciones y hechos podría ser también una estrategia válida, pero siempre y cuando actúe en compañía de otras estrategias de conflicto como son aquéllas que buscan la confrontación interna entre ideas diferentes de un mismo alumno cuando éstas entran en contradicción. De ahí que, una vez que se haya presentado el modelo científico como alternativa a la idea que sostiene el alumno a través de situaciones específicas, dediquemos suficiente atención al desarrollo de habilidades para generalizar y eliminar contradicciones (Gauld, 1988; Finegold y Gorsky, 1991). Debemos tener presente que gran parte de las dificultades del cambio en las concepciones podrían estar no sólo en la asimilación de las nuevas ideas y en el descarte de las anteriores, sino también en la capacidad de trasladar las ideas que se poseen de unos contextos a otros (Pozo, Limón y Sanz, 1991; Oliva, 1996).

En definitiva, nos parece necesario buscar fórmulas alternativas de cambio conceptual que tomen en cuenta estos distintos niveles de organización y vayan más allá de los cambios en fenómenos específicos. En este sentido, nos parece interesante la idea de potenciar nuevas fórmulas de abordar el uso de analogías como estrategia didáctica. La idea de que concepciones diferentes puedan llegar a compartir patrones de razonamiento comunes, como hemos visto en el trabajo, sugiere que la construcción de ideas científicas en unos dominios pudiera servir para trasladar esquemas de pensamiento aprendidos a otros dominios distintos. Es de esperar,

\section{REFERENCIAS BIBLIOGRÁFICAS}

ACEVEDO, J.A. (1990). Razonamiento causal en una tarea de contexto natural. Un estudio evolutivo con estudiantes de bachillerato. Investigación en la Escuela, 10, pp. 61-70.

ANDERSSON, B. (1986). The experiential gestalt of causation: a common core to pupils' preconceptions in Science. European Journal of Science Education, 8(2), pp. 155-171.

BENARROCH, A. y MARÍN, N. (1998). Dependencias de las explicaciones de los alumnos de esquemas de conocimiento específicos y generales, en Banet, E. y De Pro (eds.). Investigación e innovación en la enseñanza de las ciencias, 2, pp. 67-75. Murcia.

FINEGOLD, M. y GORSKY, P. (1991). Students' concepts of force as applied to related physical systems: a search for consistency. International Journal of Science Education, 13(1), pp. 97-113.

FURIÓ, C., y GIL, D. (1980). El programa-guía, una propuesta para la renovación de la didáctica de la física y química en el bachillerato. ICE de la Universidad de Valencia. pues, que las analogías jueguen un papel importante como intermediario en el proceso de generalización del pensamiento del alumno y de ahí la necesidad de profundizar en este terreno. Así mismo, nos parece interesante rescatar una vieja estrategia didáctica como lo es el uso de paradojas que un papel tan importante ha jugado en la evolución del conocimiento en la historia de las ciencias. Las paradojas históricas, como también las paradojas elaboradas por el profesor en sus clases, pueden constituir instrumentos interesantes de cara a conectar distintas partes del pensamiento del alumno. Se trataría de contribuir con ello a mejorar el nivel de organización de ese conocimiento, eliminando contradicciones internas y ayudando a construir un sistema conceptual más y mejor organizado.

En resumidas cuentas, nos parece interesante abordar en el futuro investigaciones en estos campos como una forma de intentar reconducir la idea de cambio conceptual recurriendo a otros procedimientos didácticos alternativos a los de confrontación directa de las ideas de los alumnos con la experiencia o con las ideas del profesor. Sólo así, pensamos, será posible poner en práctica formas de enseñanza explícitamente comprometidas con lo que es un cambio en el pensamiento de los alumnos a través de sus distintos niveles de organización.

\section{RECONOCIMIENTO}

La investigación en la que se basa este trabajo ha sido aprobada y financiada por el Ministerio de Educación y Cultura a través de la convocatoria del CIDE de ayudas a la investigación educativa.
GAULD, C.F. (1988). The congitive context of pupils' alternative frameworks. International Journal of Science Education, 10(3), pp. 267-274.

GÓMEZ-CRESPO, M.A., POZO, J.I., y SANZ, A. (1995). Students' ideas on conservation of matter: effects of expertise and context variables. Science Education, 79(1), pp. 77-93.

HIERREZUELO, J. y MONTERO, A. (1988). La ciencia de los alumnos. Madrid: Laia-MEC.

HIERREZUELO, J., et al. (1993). Ciencias de la naturaleza. física y química. Educación secundaria, $4^{\circ}$ curso. Torre del Mar: Elzevir.

LICHT, P. y THIJS, G.D. (1990). Method to trace coherence and consistence of preconceptions. International Journal of Science Education, 12(4), pp. 403-416.

MARÍN, N. (1994). Elementos cognoscitivos dependientes del contenido. Revista Interuniversitaria de Formación del Profesorado, 20, pp. 195-208. 
MONK, M. (1995). On the identification of principles in science that might inform research into students' beliefs about natural phenomena.International Journal of Science Education, 17(5), pp. 565-573.

OLIVA, J.M. (1994). «Influencia de las variables cognitivas en la construcción de conocimientos de mecánica. Un estudio empírico y un análisis computacional». Tesis doctoral no publicada. Universidad Nacional de Educación a Distancia. Madrid.

OLIVA, J.M. (1996). Estudios sobre consistencia en las ideas de los alumnos en ciencias. Enseñanza de las Ciencias, 14(1), pp. 87-92.

OLIVA, J.M.(1999a). Structural patterns in students' conceptions in mechanics. International Journal of Science Education, 21(9), pp. 903-920.

OLIVA, J.M. (1999b). Algunas reflexiones sobre las concepciones de los alumnos y el cambio conceptual. Enseñanza de las Ciencias, 17(1), pp. 93-108.

POZO, J.I. y GÓMEZ-CRESPO, M.A. (1997). ¿Qué es lo que hace difícil la comprensión de la ciencia? Algunas explicaciones y propuestas para la enseñanza, en Del Carmen, L. (comp.). La enseñanza y el aprendizaje de las ciencias de la naturaleza en la educación secundaria. Madrid: Horsori.
POZO, J.I. y GÓMEZ-CRESPO, M.A. (1998). Aprender y enseñar Ciencias. Madrid: Morata.

POZO, J.I., GÓMEZ CRESPO, M.A. y LIMÓN, M. y SANZ, A. (1991). Procesos cognitivos en la comprensión de la ciencia: las ideas de los adolescentes sobre la química. Madrid: CIDE.

POZO, J.I., PÉREZ, M.P., SANZ, A. y LIMÓN, M. (1992). Las ideas de los alumnos sobre la ciencia como teorías implícitas. Infancia y Aprendizaje, 57, pp. 3-22.

POZO, J.I., LIMÓN, M. y SANZ, A. (1991). Conocimientos previos y aprendizaje escolar. Cuadernos de Pedagogía, 188, pp. 12-14.

TOUGER, J., DUFRESNE, J., GERACE, W., HARDINAN, P. y MESTRE, J. (1995). How novice physics students deal with explanations. International Journal of Science Education, 17(2), pp. 255-269.

VIENNOT, L. (1985). Analysing students' reasoning in science: a pragmatic view of theoretical problems. European Journal of Science Education, 7(2), pp. 151-162.

VOSNIADOU, S. (1994). Capturing and modelling the process of conceptual change. Learning and Instruction, 4(1), pp. 45-69. 


\section{INVESTIGACIÓN DIDÁCTICA}

\section{Apéndice}

Cuestión número 2 del cuestionario A

Lanzamos verticalmente hacia arriba con la misma velocidad una bola de madera de 50 gramos y otra de acero de 500 gramos.

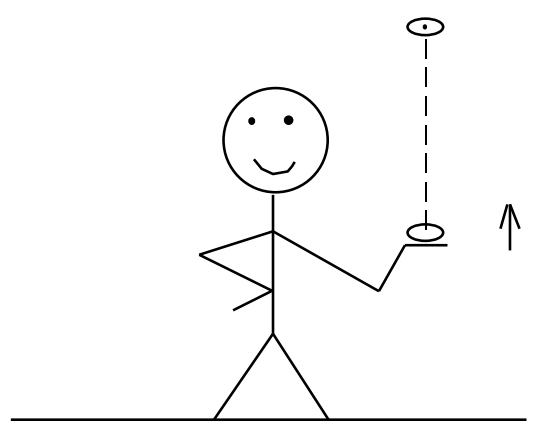

Despreciando todo efecto de rozamiento con el aire, señala qué te parecen las siguientes afirmaciones en referencia a cuál es la piedra que se para y deja antes de subir:

a) Se para antes la bola de madera (50 gramos), que es la que será frenada más fácilmente por la gravedad.

$\begin{array}{lrcccl}-3 & -2 & -1 & 0 & +1 & +2\end{array}$

b) Se para antes la bola de acero (500 gramos), que es la que más pesa.

$\begin{array}{lrccll}-3 & -2 & -1 & 0 & +1 & +2 \\ \text { Desacuerdo total } & & \text { Indeciso } & & & \text { Acuerdo total }\end{array}$

c) Ambas se paran al mismo tiempo. La masa no influye siempre que no haya rozamiento con el aire.

$\begin{array}{lrcccl}-3 & -2 & -1 & 0 & +1 & +2\end{array}+3$

